\title{
Stabilisation appliance improves short term outcomes in TMD
}

\author{
Ekberg E, Vallon D, Nilner M. Occlusal appliance therapy in patients with temporomandibular disorders. A double \\ blind controlled study in a short-term perspective. Acta Odontol Scand 1998; 56:122-128.
}

\begin{abstract}
Objective To investigate the short-term effect of treatment with a stabilisation appliance compared with a control appliance in patients of arthrogenous origin.
\end{abstract}

Design A double blind randomised controlled trial

Intervention 66 patients fulfilling the entry criteria had either a stabilisation appliance fitted which had a smooth flat surface with all supporting teeth in contact, or a control appliance, which did not alter the inter-maxillary relationship. Initial screening, history, clinical examination and final assessment was carried out by one specialist, while the treatments were provided by another. Treatments were evaluated after 10 weeks. In additional to a clinical examination before and after questionnaires were completed by the patients.

Outcome measures Frequency and intensity of the pain were assessed using the questionnaire and a visual analogue scale. A number of clinical signs were also recorded - maximal opening, pain during mandibular movements, muscle and joint tenderness.

\section{Commentary}

The placebo effect is a feature of any treatment offered for temporomandibular disorders. Green and Laskin illustrated this in $1972^{1}$. There have been uncontrolled studies illustrating the benefits of occlusal splint therapy but most have little meaning as the placebo effect has not been considered. This paper illustrates a pains-taking technique to overcome criticisms of past papers and uses a rigorous patient selection technique to recruit patients with near identical conditions. It then processed the patients along two different paths of treatment using measurable objective outcome parameters. The trial was blinded to the observers but not to the patients - thus it was an open observerblind trial, not a double-blind trial.

There are difficulties with trials on patients with TMJ disorders and particularly where it is hoped that patients will be purely myogenous or purely arthrogenous in their symptoms and signs. This
Results 33 different outcome measures are reported; 7 achieved statistical significance. Three are shown in the table below. Calculating the NNT for each of these three measures shows that you only need to treat 3 patients with the stabilisation appliance for one additional patient to feel better (subjectively), have reduced daily or constant pain and lateral tenderness.

\begin{tabular}{|c|c|c|c|c|}
\hline & Test group & Control Group & $\begin{array}{l}\text { Odds Ratio } \\
\text { (95\% Cl) }\end{array}$ & $\begin{array}{c}\text { NNT } \\
(95 \% \mathrm{Cl})\end{array}$ \\
\hline $\begin{array}{l}\quad n \\
\text { Daily or } \\
\text { constant pain }\end{array}$ & $\begin{array}{l}30 \\
10\end{array}$ & $\begin{array}{l}30 \\
19\end{array}$ & $0.28(0.09-0.8)$ & $3 \mid 2-17$ \\
\hline $\begin{array}{l}\text { Symptom -free } \\
\text { better (subjective }\end{array}$ & 25 & 15 & $4.0(1.48-16)$ & $3(2-9)$ \\
\hline $\begin{array}{l}\text { Lateral TMJ } \\
\text { tenderness }\end{array}$ & 9 & 18 & $0.28(0.09-0.8)$ & $3 \mid 2-17$ \\
\hline
\end{tabular}

Conclusion The authors conclude that both stabilisation and control appliance had an effect on TMD pain. There was a more statistically significant effect in the stabilisation group.

Address for reprints: EwaCarin Ekberg, Centre for Oral Health Sciences, Carl Gustafs v34, S-21421 Malmo, Sweden paper concentrates on those with arthrogenous TMD and attempts to exclude the myogenous. It is impossible to do this as illustrated by the finding of muscle tenderness in more than 4 muscle sites in $93 \%$ of patients. This did not change in either group with treatment although more treatment group patients had less tenderness of the TMJ. There was subjective improvement in both groups, but more in the treatment group.

Other difficulties in designing trials in TMJ patients lie in the multitude of variables within the patient groups that could influence outcome. Clinical experience suggests that those with a long history require longer treatment. Equally, the psychological background of patients is important. ${ }^{2}$ Neither of these factors has been considered by this paper.

The thoroughness of this trial is exemplary and the results do seem to indicate that the stabilisation splint has some advantage over the placebo. However, as the authors themselves state, the true outcome of treatment cannot be seen at 10 weeks. A longer perspective is required.

For management of patients with TMJD now, the evidence is that appliances help many patients. Thus the pragmatic approach would be to construct a stabilisation splint from meticulous impressions and occlusal records prepared by the dentist and to fit it without taking up too much surgery time. If it fails to improve symptoms, then it should be adjusted to conform exactly with the principles of the stabilisation splint. The psychological background lifestyle of the patients must always be addressed before any other treatment is undertaken.

1 Green C S, Laskin D M. Splint therapy for the myofascial pain-dysfunction (MPD) syndrome. I Am Dent Assoc 1972; 84: 634-628

2 Gatchel R J, Garofalo J P, Ellis C H. Major psychological disorders in acute and chronic TMD. J Amer Dent Assoc 1996; 127: 1365-1374

R P Juniper

University of Oxford, Oxford, UK 